\section{Long-term outcome of conjunctival fixation sutures to the sclera for prolapsed subconjunctival orbital fat}

\begin{abstract}
Purpose To report long-term outcome of new surgical technique for prolapsed subconjunctival orbital fat.

Patients and methods Retrospective study was conducted on 48 eyes of 37 patients who underwent excision of prolapsed subconjunctival orbital fat with conjunctival fixation to the sclera. Complications and recurrence were evaluated.

Results The mean follow-up period was 39 months (range, 8-101 months). Two eyes (4.4\%) developed recurrence at 4 and 8 years after surgery. No long-term complication was found.

Conclusions The new surgical technique to manage prolapsed subconjunctival orbital fat using conjunctival fixation to the sclera was very useful and effective, with few recurrence and no long-term complication.

Eye (2017) 31, 749-752; doi:10.1038/eye.2016.302; published online 20 January 2017
\end{abstract}

\section{Introduction}

Subconjunctival orbital fat prolapse is a yellow, round protruded lesion in the superotemporal conjunctiva, which is herniated intraconal orbital fat through a weakened Tenon's capsule. ${ }^{1,2}$ This process is analogous to the herniation of extraconal orbital fat into the eyelids through weakened orbital septum, which is frequently seen in elderly persons. ${ }^{3,4}$

Surgical treatment is needed when the lesion is cosmetically unacceptable or causes irritation during eyelid closure. ${ }^{5}$ Transconjunctival fat excision is the simple technique ${ }^{1,2,6}$ with a recurrence rate as high as 9\%. ${ }^{7}$ Otaka and $\mathrm{Kyu}^{5}$ reported surgical technique of conjunctival fixation to the sclera without fat excision in a small number of patients; however, pushing
MK Yang ${ }^{1,2}$, N Kim ${ }^{1}, \mathrm{H}-\mathrm{K}$ Choung $^{3}$

and SI Khwarg ${ }^{2}$

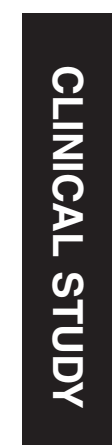

herniated fat posteriorly without volume reduction requires extensive effort and its longterm outcome was not evaluated. Therefore, we have proposed a new surgical technique in which transconjunctival fat excision is combined with conjunctival fixation to the sclera. In this study, we describe this technique for prolapsed subconjunctival orbital fat and report the longterm surgical outcomes.

\section{Materials and methods}

This study retrospectively reviewed the medical records of 37 patients who had undergone surgical correction for prolapsed subconjunctival orbital fat by excision with conjunctival fixation to the sclera technique between November 2002 and June 2012, in three different hospitals. Using electronic medical records and medical photographs, we collected data on sex, age at diagnosis, laterality and location of the lesion (Figure 1). Complications and recurrence were reviewed.

We also evaluated prevalence of eyelid fat prolapse to investigate analogy between eyelid fat prolapse and subconjunctival fat prolapse; age-matched controls were recruited and prevalence of eyelid fat prolapse was compared between two groups.

Surgery was performed by one of three oculoplastic surgeons, all of whom used the same surgical technique of transconjunctival fat excision with conjunctival fixation to the sclera. Detailed surgical technique is illustrated in Figure 2. Briefly, the superotemporal conjunctiva was incised circumferentially and the prolapsed orbital fat was partially excised. Conjunctivaclosing interrupted sutures were applied with 6-0 Vicryl (polyglactin 910; Ethicon Inc., Somerville, NJ, USA) which concurrently engaged the underlying episcleral tissue, to
${ }^{1}$ Department of Ophthalmology, Seoul National University College of Medicine, Seoul National University Bundang Hospital, Seongnam, Korea

${ }^{2}$ Department of Ophthalmology, Seoul National University College of Medicine, Seoul National University Hospital, Seoul, Korea

${ }^{3}$ Department of Ophthalmology, Seoul Metropolitan GovernmentSeoul National University Boramae Medical Center, Seoul, Korea

Correspondence: N Kim, Department of Ophthalmology, Seoul National University Bundang Hospital, \#300 Gumi-dong, Bundang-gu, Gyeonggi-do, Korea Tel: +82 31787 7376; Fax: +82 317874057 E-mail: resourceful@ hanmail.net

Received: 29 June 2016 Accepted: 20 November 2016;

Published online:

20 January 2017 Seongnam 463-707, 

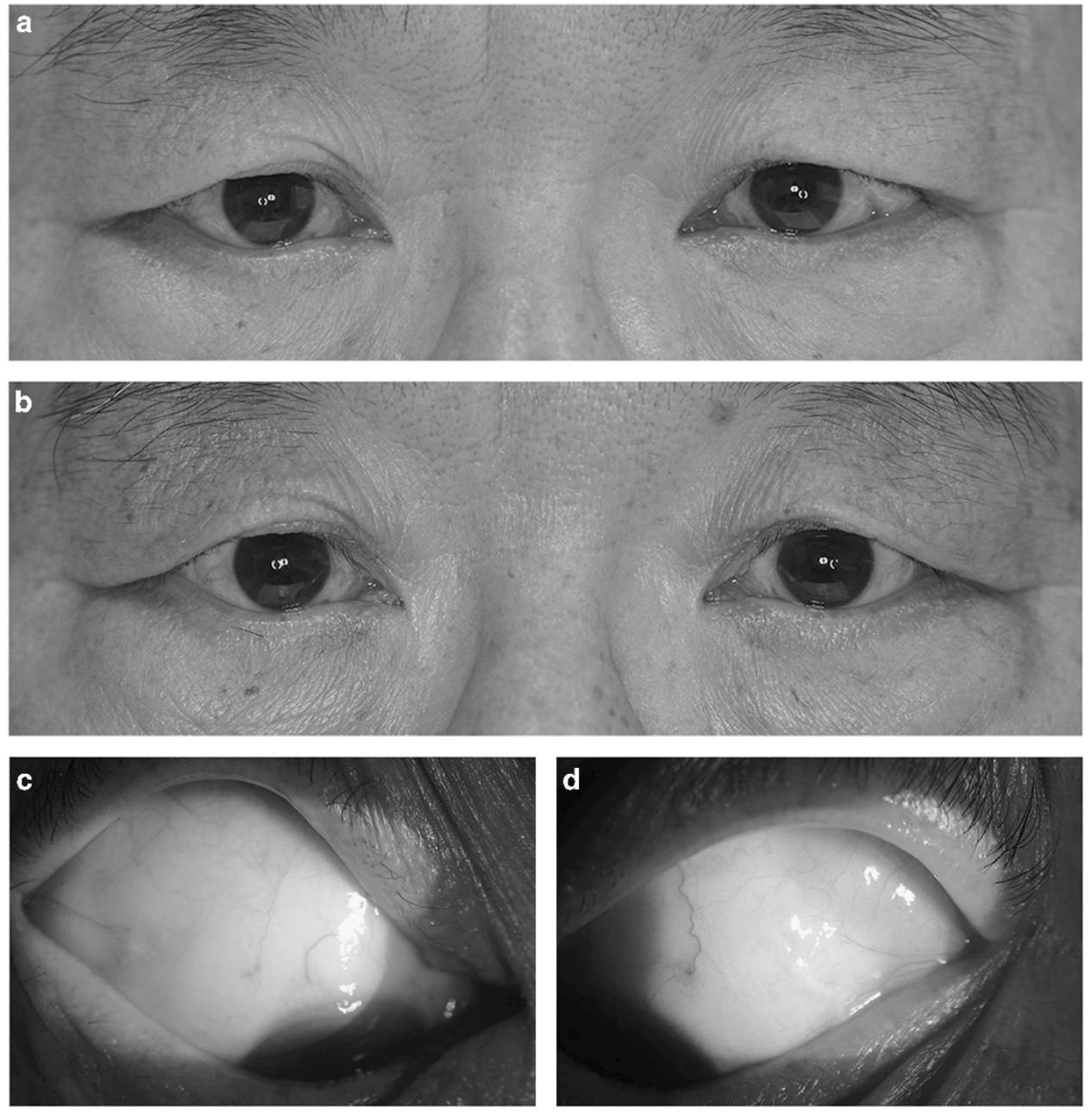

Figure 1 Photograph of a patient who underwent transconjunctival fat excision with the conjunctival fixation to the sclera technique. Preoperatiely, prolapsed subconjunctival fat was visible in the primary gaze (a). Five years after the surgery, fat was not visible without recurrence or complications (b). Note the well-sustained barrier effect at the surgical scar (c, d).

serve as a barrier. The excised fat was evaluated volumetrically and pathologically in several cases.

Patients were followed up at 1 week, 2, 6 months after surgery and further, with slit-lamp biomicroscopic examination and photographic recording. Recurrence was defined as the presence of a noticeable lesion at primary gaze or downgaze. Statistical analysis was performed by using SPSS (version 18.0, SPSS Inc.). Nominal variables were compared by using Fisher's exact test. The institutional review board of the Seoul National University Bundang Hospital approved the study prospectively.

\section{Results}

The mean age of 37 patients at diagnosis was 64.4 years, with males predominating (male:female $=29: 8$ ). Most of the subconjunctival fat prolapse was located in superotemporal area (46 eyes, 95.8\%), followed by 1 in inferonasal and 1 in nasal area. Eyelid orbital fat prolapse was more frequently found in patients with subconjunctival orbital fat prolapse than in control group (35/44 vs. 22/44, Fisher's exact test, $p=0.007)$.

Among 37 patients of 48 eyes who underwent surgical correction, mean postoperative follow-up period was 39 months (range, 8-101 months). During long-term follow-up, two eyes (4.4\%) developed late recurrence at 4 and 8 years after surgery. Postoperative complications were found only in two cases; severe chemosis and a conjunctival cyst. Both complications were spontaneously resolved within a month and further treatment was not required. 

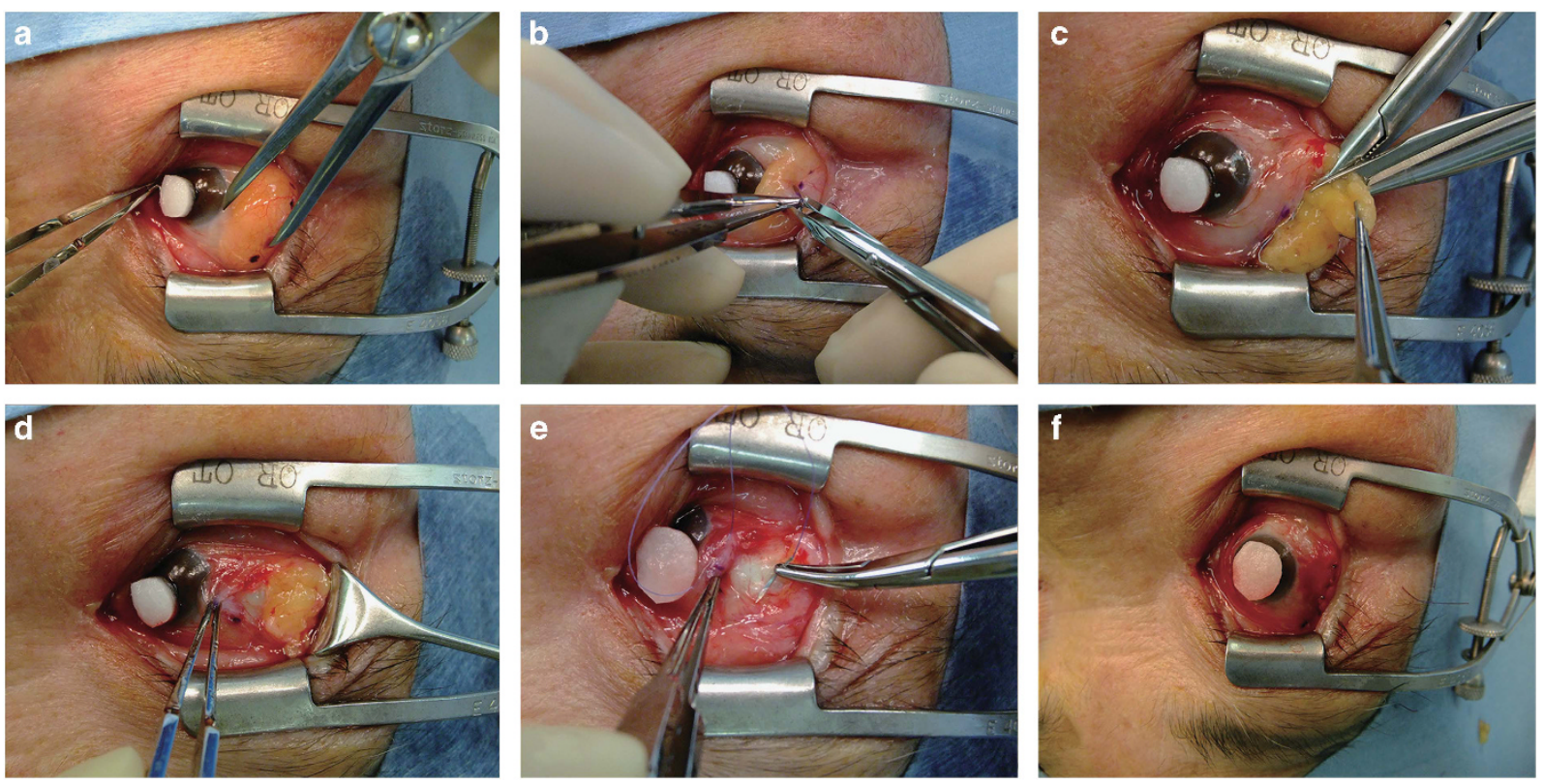

Figure 2 Transconjunctival excision of prolapsed subconjunctival orbital fat with the conjunctival fixation to the sclera technique. The mark for the superotemporal conjunctival incision was made circumferentially at $8 \mathrm{~mm}$ from the limbus (a). Following lidocaine injection, the conjunctival incision was made by using Westcott scissors along the mark (b). After incision of Tenon's capsule, the prolapsed orbital fat was exposed and partially excised (c). The remnant fat was pushed back to its original position (d). Conjunctivaclosing interrupted sutures were applied, which engaged the underlying episcleral tissue while 6-0 Vicryl was used to serve as a barrier (e). Postoperative view (f).

\section{Discussion}

In this study, the new surgical technique with conjunctival fixation to the sclera showed lower recurrence rate of $4 \%$ during longer follow-up period compared with simple transconjunctival fat excision.? Moreover, these recurrences occurred later (51 and 101 months) compared with the other surgical technique (40, 47 and 52 months). ${ }^{7}$ Recurrent cases showed clinically acceptable outcomes and the patients were satisfied without further management.

Our surgical technique involves conjunctiva-closing sutures engaging episcleral tissue, which provides barrier effect against protruding orbital fat without additional surgical time or technical difficulty. There were no conjunctival complications such as a visible conjunctival scar and extraocular muscle restriction either.

We also found that, for the first time, prolapsed eyelid fat was found more frequently in patients with subconjunctival orbital fat prolapse compared with agematched control group. This finding implies that weakness of Tenon's capsule is associated with concurrent weakness of orbital septum.

In conclusion, we introduced a new surgical technique of transconjunctival fat excision with conjunctival fixation to the sclera, and reported its good surgical outcome.

\section{Summary}

What was known before
In most cases, subconjunctival orbital fat prolapse
is found in superotemporal bulbar conjunctiva of
elderly person. Surgical treatment is required for
cosmetic unacceptability or irritability during eyelid
closure. Simple transconjuntival fat excision is a
frequently used technique. Conjunctival fixation to
the sclera without fat excision was also showed good
result in a case series.
What this study adds
Transconjunctival fat excision combined with conjunctival
fixation to the sclera showed lower recurrence in long-
term follow-up. Adding barrier suture is a simple and
effective technique to obtain better surgical outcomes.

\section{Conflict of interest}

The authors declare no conflict of interest.

\section{Acknowledgements}

This research received no specific grant from any funding agency in the public, commercial or not-for-profit sectors. 


\section{References}

1 Glover AT, Grove AS Jr. Subconjunctival orbital fat prolapse Ophthal Plast Reconstr Surg 1987; 3: 83-86.

2 Jordan DR, Tse DT. Herniated orbital fat. Can J Ophthalmol 1987; 22: 173-177.

3 Kim YD, Goldberg RA. Orbital fat prolapse and dermolipoma: two distinct entities. Korean J Ophthalmol 1994; 8: $42-43$.
4 Jordan DR. Orbital fat prolapse. Arch Ophthalmol 1993; 111: 1583.

5 Otaka I, Kyu N. A new surgical technique for the management of orbital fat prolapse. Am J Ophthalmol 2001; 131: 267-269.

6 McNab AA. Subconjunctival fat prolapse. Aust N Z J Ophthalmol 1999; 27: 33-36.

7 Siban M, Weijtens O, van den Bosch W, Paridaens D. Efficacy of transconjunctival excision of orbital fat prolapse: a longterm follow-up study. Acta Ophthalmol 2014; 92(3): 291-293. 